\title{
Modes de Lamb de deux plaques élastiques couplées par une couche d'eau
}

\author{
O. LENOIR, H. KHELIL, J.-L. IZBICKI, M. ROUSSEAU*, F. COULOUVRAT* et \\ Ph. GATIGNOL**
}

Laboratoire d'Acoustique Ultrasonore et d'Electronique, Université du Havre, URA 1373 du CNRS, Place R. Schuman, 76610 Le Havre, France

* Laboratoire de Modélisation en Mécanique, Université Pierre et Marie Curie, URA 229 du CNRS, Case 162, 4 Place Jussieu, 75252 Paris cedex 05, France

${ }^{* *}$ LG2mS, Université de Technologie de Compiègne, URA 1505 du CNRS, BP. 206, 60206 Compiègne cedex, France

\begin{abstract}
A comparison theory / experiment describes the behaviour of acoustical free modes for a structure composed of two identical elastic layers coupled by a thin water film. The solutions of the dispersion equation written under a closed form, are obtained numerically. Simultaneously, the high and low frequence limits, and also the cut-off frequencies are evaluated asymptotically. The cut-off frequencies repartition has been confirmed by the experiment, on either the retrodiffusion or the resonance spectrum.
\end{abstract}

\section{INTRODUCTION.}

Les modes propres de vibration d'une structure composée d'un assemblage de couches fluides ou solides, sont largement étudiés en prospection sous-marine, ou en contrôle non-destructif [1] , [2]. Il est souvent difficile de décrire le comportement des solutions ainsi obtenues, aussi est-il intéressant de les comparer à des modes plus connus comme les modes de Lamb [3]. L'objectif est d'étudier la structure particulière constituée par l'assemblage Vide / S / F / S / Vide, où le fluide (F) est une fine couche d'eau assurant le couplage entre deux plaques élastiques identiques (S).En particulier, le dédoublement des fréquences de coupure des modes de Lamb purs prédit par la théorie, est confirmé par l'étude expérimentale.

\section{ETUDE ANALYTIQUE.}

\subsection{Relation de dispersion}

On considère deux couches solides identiques, d'épaisseur $2 \mathrm{~h}$, constituées par un même milieu élastique de masse volumique $\rho, \quad c_{L}$ et $c_{T}$ étant respectivement les vitesses des ondes longitudinales et transversales. On note $\rho_{F}$ la masse volumique de l'eau, $\mathrm{c}$ la célérité du son et d l'épaisseur de la couche assurant le couplage. En écrivant les conditions de continuité à chaque interface, on obtient l'équation de dispersion écrite sous forme semi-factorisée, pour la structure V/ S / F / S / V, (avec les notations de [3 ]).:

(1) $\mathrm{D}\left(\omega, k_{x}\right)=4 \tau C_{a} C_{s}\left(C_{a}-C_{s}\right) \cos \left(k_{Z F} d_{F}\right)+\left(4 C_{a}^{2} C_{s}^{2}-\tau^{2}\left(C_{a}-C_{s}\right)^{2}\right) \sin \left(k_{Z F} d_{F}\right)$

\subsection{Etude à haute et basse fréquence}

L'étude des solutions réelles de Eq. (1) montre qu'il existe, à basse fréquence, quatre modes propres. Deux d'entre eux $\left(S_{0}^{I}\right.$ et $\left.S_{0}^{I I}\right)$ ont la même limite que le mode de Lamb pur $S_{0}$; un autre (noté $\left.A_{0}^{\prime}\right)$ a le même comportement que le mode de Lamb pur $A_{0}$; le dernier mode (noté $F_{0}$ ) dépend fortement de la couche 
fluide (Fig. 1). A haute fréquence, on montre que deux modes tendent vers londe de Scholte-Stoneley F / S et deux vers l'onde de Rayleigh V / S, correspondant à chacune des interfaces de la structure. Les autres modes propres se comportent à haute fréquence, soit comme des modes de Lamb purs dans une plaque, soit comme des modes guidés dans la couche fluide (ce dernier cas uniquement si $c<c_{T}$ ).

\subsection{Etude des fréquences de coupure}

Elles sont solutions de $D\left(\omega, k_{x}=0\right)=0$.

On distingue deux familles de modes : ceux qui, à leur fréquence de coupure, sont purement transversaux (notés " $T$ ") et ceux qui, à leur fréquence de coupure, sont purement longitudinaux (" $L$ ").

- Les ondes transversales n'étant pas transmises par la couche d'eau, les fréquences de coupure des modes $T$ coïncident avec celles des modes de Lamb purs de même type. Par ailleurs, ces solutions sont racines doubles (une pour chaque couche).

- Les ondes longitudinales étant transmises par le fluide, les deux couches élastiques sont alors couplées. Cet effet de couplage dépend essentiellement : (i) du contraste d'impédance S / F; (ii) de l'épaisseur relative de la couche d'eau.

L'étude analytique montre qu'il existe deux sous-familles, notées "+" et "-", de modes "L", dont les fréquences de coupure sont respectivement solutions de :

$$
L_{+}=\left(k_{L} h\right)\left(\operatorname{tg}\left(k_{L} h\right)-\cot g\left(k_{L} h\right)\right) \approx \eta=4 \frac{\rho}{\rho_{F}} \frac{h}{d}
$$

$$
L_{-}=\frac{\operatorname{tg}\left(k_{L} h\right)-\cot g\left(k_{L} h\right)}{k_{L} h} \approx v=\frac{\rho}{\rho_{F}}\left(\frac{c_{L}}{c_{F}}\right)^{2} \frac{d}{h}
$$

- Dans le cas de la sous-famille "+", Eq. (2), pour une couche d'eau mince et un fort contraste d'impédance, les phénomènes (i) et (ii) agissent dans le même sens $(\eta \gg>1)$. Les racines sont alors pratiquement les fréquences de coupure des modes de Lamb purs " $L$ ".

- Dans le cas de la sous-famille "-", Eq. (3), les phénomènes (i) et (ii) agissent en sens contraire.

(a) Lorsque (i) prédomine sur (ii) ( $v>>1)$, le couplage est faible, et on trouve à nouveau les fréquences de coupure des modes de Lamb purs " $L$ ".

Dans ce cas, les fréquences de coupure " $L+$ " et " $\mathrm{L}$ " sont donc chacune très voisines de celles des modes de Lamb purs; aussi observera-t-on un dédoublement de ces modes (Fig. 1).

En dessous de toutes ces fréquences, il existe en outre une solution supplémentaire (racine simple) correspondant à un mode appelé vertical (V, Fig.1). La fréquence de coupure de ce mode dépend fortement de la valeur du paramètre $v$.

(b) Lorsque (ii) prédomine sur (i) $(v<1)$, on est ramené au problème de deux plaques élastiques en glissement lune sur l'autre. Les fréquences de coupure "L-" sont alors celles d'une plaque en appui simple sur un substrat rigide.

\section{ETUDE EXPERIMENTALE}

L'étude expérimentale est réalisée avec des plaques d'ahuminium $\mathrm{A}\left(\rho=2800 \mathrm{~kg} / \mathrm{m}^{3}, c_{L}=6380 \mathrm{~m} / \mathrm{s}\right.$, $c_{T}=3100 \mathrm{~m} / \mathrm{s}$ ) de $5 \mathrm{~mm}$ d'épaisseur, placées verticalement dans l'eau. La plaque seule $A$, ou la structure multicouche A/E/A comprenant deux plaques A séparées par une fine couche d'eau $E$ d'épaisseur de l'ordre de $0.1 \mathrm{~mm}$, sont étudiées en incidence normale par une méthode impulsionnelle monostatique. A cet effet, on utilise un transducteur de fréquence centrale $2,25 \mathrm{MHz}$, de bande passante efficace comprise entre 500 $\mathrm{kHz}$ et $3.5 \mathrm{MHz}$, excité par des impulsions électriques. Le signal réfléchi est reçu par le même transducteur, puis envoyé sur un oscilloscope numérique où il est analysé par un algorithme de transformée 
de Fourier. Si on traite la totalité du sigual réfléchi, on obtient un spectre de rétrodiffusion,; si on enlève l'écho de réflexion spéculaire, on obtient un spectre de résonances.

Une plaque A seule est d'abord étudiée pour déterminer les fréquences des modes de Lamb effectivement excités. Ces modes sont repérés par des minima du spectre de rétrodiffusion (Fig.2), et par des pics sur le spectre de résonance (Fig.3). Bien que l'on soit en incidence normale, les effets de diffraction liés à la surface finie de la pastille émettrice du transducteur excitent quelques ondes transversales liées aux modes symétriques $\mathrm{S} 1$ et $\mathrm{S} 4$ (modes transversaux T2 et T6). Cependant, on observe principalement les modes longitudinaux symétriques S2, S5 et S8 (modes L1, L3 et L5), et les modes antisymétriques A3 et A6 (modes $\mathrm{L} 2$ et $\mathrm{L} 4$ ), correspondant aux creux larges du spectre de rétrodiffusion, et aux pics larges du spectre de résonances.

Pour la structure $\mathrm{A} / \mathrm{E} / \mathrm{A}$, on retrouve sur le spectre de rétrodiffusion (Fig.4) des creux larges aux fréquences d'excitation des modes longitudinaux S2, A3, S5, A6 et S8, et des creux plus fins liés aux modes transversaux S1, S4 et S7 (Tab.1). On observe en outre, à proximité des modes A3, S5, A6 et S8 (modes longitudinaux L2, L3, L4 et L5), d'autres creux larges correspondant à l'excitation de modes plutôt longitudinaux, notés $A 3^{+}, S 5^{+}, A 6^{+}$et $S 8^{+}$. Sur le spectre de résonances (Fig.5), on observe nettement deux pics larges au voisinage de chacune des fréquences d'excitation des modes $A 3, \mathbf{S} 5$ et $A 6$ de la plaque seule. On confirme ainsi expérimentalement le dédoublement des modes longitudinaux pour une structure $\mathbf{A} / \mathbf{E} / \mathrm{A}$.

Pour une structure périodique à $n$ motifs $\mathrm{A} / \mathrm{E}$ insonée à incidence normale, on met en évidence des résonances liées à certains modes de Lamb longitudinaux de la plaque A seule; pour chacun de ces modes, on peut observer ( $\mathrm{n}-1)$ résonances supplémentaires associées.

Tab.1 :

\begin{tabular}{|c|c|c|c|c|c|c|c|c|c|c|c|}
\hline Mode & $\mathrm{S} 2$ & $\mathrm{~A} 3$ & $\mathrm{~S} 1$ & $\mathrm{A3}^{+}$ & $\mathrm{S} 4$ & $\mathrm{~S}^{+}$ & $\mathrm{S} 5$ & $\mathrm{A6}^{+}$ & $\mathrm{A6}^{+}$ & $\mathrm{S}^{+}$ & $\mathrm{S} 8$ \\
\hline L ou T & $\mathrm{L} 1$ & $\mathrm{~L} 2$ & $\mathrm{~T} 2$ & & $\mathrm{~T} 6$ & & $\mathrm{L3}$ & $\mathrm{L} 4$ & & & L5 \\
\hline Fréquence théorique $(\mathrm{MHz})$ & 0,638 & 1,276 & 0,620 & & 1,860 & & 1,914 & 2,552 & & & 3,190 \\
\hline Rétrodiffusion $(\mathrm{MHz})$ & 0,641 & 1,261 & 0,601 & 1,311 & & 1,865 & 1,895 & 2,520 & 2,535 & 3,120 & 3,155 \\
\hline Résonance $(\mathrm{MHz})$ & 0,650 & 1,260 & 0,605 & 1,320 & & 1,860 & 1,908 & 2,512 & 2,534 & 3,115 & 3,159 \\
\hline
\end{tabular}

\section{Références :}

[1] H.Franklin, "Propagation des ondes d'interface dans les milieux à plusieurs couches. Application à certains modèles de fonds marins", Thèse de l'Université Paris 6, 1991

[2] A.K Mal, P.C. Xu \& Y. Bar-Cohen, Int. J. Engng. Sci., 27, pp. 779-791, 1989

[3] R. Fiorito, W. Madigosky, and H. Überall, "Resonance theory of acoustic waves interacting with an elastic plate", J. Acoust. Soc. Am., 66, pp. 1857-1866,1979 


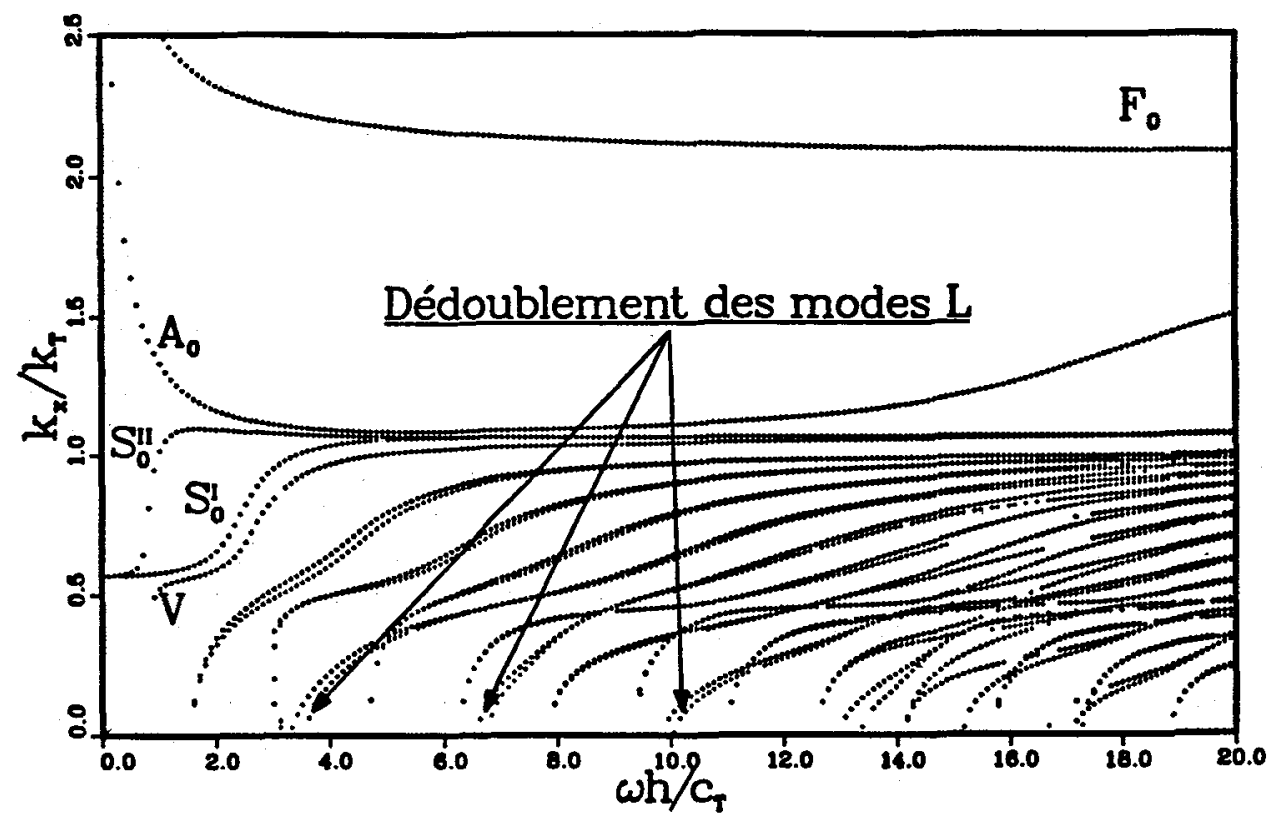

Figure 1 : Modes propres d'une structure $A l u / E a u / A l u ~(d / h=0,1)$

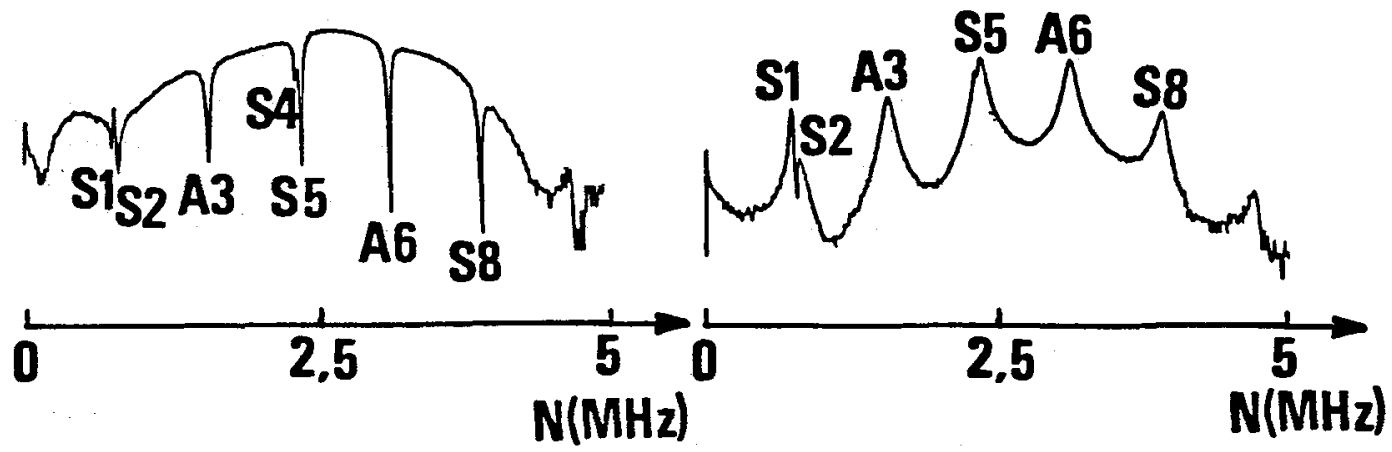

Figure 2 : Spectre de rétrodiffusion de la plaque $A$
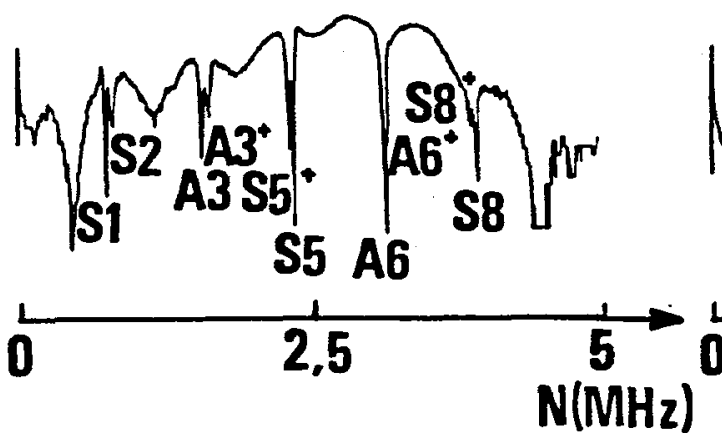

Figure 4 : Spectre de rétrodiffusion de la structure AEA
Figure 3 : Spectre de résonances de la plaque $A$

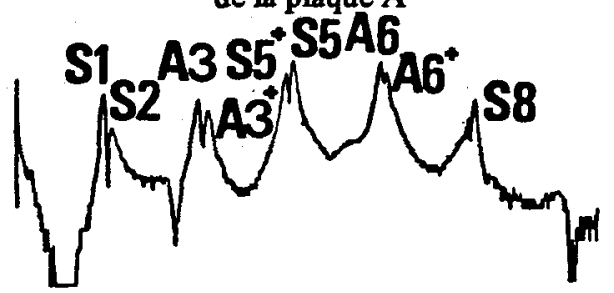

